\title{
IMPROVING THE REFERENCE NETWORK IN WIDE-AREA PERSISTENT SCATTERER INTERFEROMETRY FOR NON-URBAN AREAS
}

\author{
Kanika Goel, Nico Adam, Robert Shau and Fernando Rodriguez-Gonzalez \\ Remote Sensing Technology Institute (IMF), German Aerospace Center (DLR), Germany
}

\begin{abstract}
Advanced Interferometric SAR (InSAR) technique, namely, Persistent Scatterer Interferometry (PSI), allows long term deformation time series analysis with millimeter accuracy. Reference network arcs construction, arcs estimation and integration for PSs are an important step in PSI. In rural regions, low density of PSs leads to separate clusters during reference network construction. Also, in case of wide-area PSI using ERS-1/2 or Sentinel-1 data, the computational load can be very high. Due to this, the reference network processing is usually divided into overlapping blocks and merged later. This can however lead to spatial error propagation. This paper presents algorithms for improving the reference network in wide-area PSI, with a focus on non-urban areas.
\end{abstract}

Index Terms-Deformation Monitoring, ERS-1/2, Interferometric SAR (InSAR), Non-Urban Areas, Persistent Scatterer Interferometry (PSI), Reference Network, Sentinel-1.

\section{INTRODUCTION}

Interferometric SAR (InSAR) is a powerful technique to measure Earth's topography and surface displacements [1], [2]. Various InSAR stacking techniques have been developed that utilize a stack of SAR images of the same area, taken at different times, for deformation time series analysis [3]-[7]. Persistent Scatterer Interferometry (PSI) exploits phase-stable Persistent Scatterers (PSs) and provides a parametric estimation of the displacement and 3D location [3], [4]. It consists of four main steps as shown in Fig. 1.

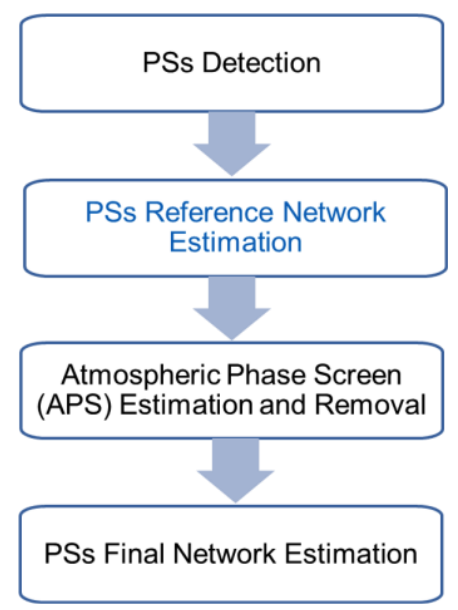

Figure 1: Main steps in PSI technique.
First, differential interferograms with respect to a single master image are formed and PSs (i.e. pixels exhibiting a low phase dispersion) are detected in the scene based on normalized amplitude dispersion index or signal-to-clutter ratio. Second, a reference network is established on the initial set of PS candidates that are of high quality. These PS candidates are linked by arcs and a reference point is assigned for the reference network. Differential arcs estimation is performed, followed by integration for PSs with respect to the reference point. Third, the atmospheric phase components are estimated for the initial PS candidates. An interpolation is then performed to estimate and remove the APS for each interferogram. Finally, all the remaining PS candidates are selected and estimated relative to the closest PS of the reference network. The quality of the PS analysis depends on the number of available images and the density of PSs. A minimum of about 25 images and a PS density of approximately 5-10 PS/km2 are recommended.

SAR missions such as ERS-1/2 and Sentinel-1 provide a continuous and large coverage at conventional resolution. Although PSI has been successfully demonstrated and validated for various applications, there are some limitations for processing a large-scale dataset. First, PSI is most effective in urban areas that have a large number of stable scatterers. For large-scale PSI, even non-urban areas need to be processed; and these are characterized by low density of PSs, their inhomogenous spatial distribution, phase ambiguities and atmospheric effects. Robust algorithms are thus required for scatterer selection, reference network estimation, and atmospheric phase removal. Second, the computational load can be very high, due to which, the processing is usually divided into overlapping blocks and merged later. This can however lead to spatial error propagation.

This paper presents algorithms that have been developed for a robust PSI reference network estimation, while mitigating error propagation. Focus is on reference network construction and inversion for wide-area PSI incorporating rural regions.

\section{METHODOLOGY}

This section explains the algorithms that have been developed to improve the following sub-steps of the PSI technique:

\subsection{Reference Network Construction}

PSI reference network is a redundant network, wherein, each high quality PS is connected to its stable neighboring PSs 
based on an exhaustive spatial search. Typically, only points that are closer than a certain distance are linked in order to cancel out atmospheric effects. However, in non-urban areas, low density of PSs leads to separate clusters in the reference network. Long arcs can be useful in such cases as long as they satisfy the quality criterion i.e. the temporal coherence.

An improved reference network can be constructed as follows:

- Divide the reference network PSs in the scene into different clusters based on $k$-means clustering. Initial cluster centers can be assigned based on prior knowledge of cluster distance for an area (e.g. distance between villages in Europe is around $1-2 \mathrm{~km}$ ).

- Form a network of these clusters based on tuning parameters: Maximum range and maximum number of arcs per cluster.

- For every triangular cycle of network of clusters, form arcs within a cluster and cluster-to-cluster, enforcing spatial homogenity based on quadrants. The tuning parameters are: Number of PSs per cluster, maximum range and maximum number of arcs per PS.

- Discard duplicate arcs.

- Check for arcs quality and visualize the reference network.

\subsection{Reference Network Integration}

In wide-area PSI, the relative deformation and residual DEM are estimated for the reference network arcs using the LAMBDA estimator. The relative measurements of the network are finally integrated via least-squares inversion. Instead of inverting a large system of linear equations (e.g. $10,000,000$ arcs for 500,000 scatterers is a typical configuration for Sentinel-1), the scene is conventionally divided into overlapping blocks that are processed separately from the beginning and merged later. However, if the inversion of a large system can be achieved somehow, then there would not be spatial error propagation due to block processing.

Network inversion involves solving a system of linear equations given by: $A x=b$, where, $A$ is the matrix which defines the reference network arcs connecting the PSs, $b$ is the vector of observations (differential deformation or differential topographic error of the arcs), and $x$ is the vector of unknowns (deformation or topographic error of the PSs wrt the reference PS. For inverting a large single-network, the following strategies can be adopted:

- Instead of solving: $A x=b$, solve for: $A^{T} W A x=A^{T} W b$, where $W$ is the covariance matrix of the observations.

- $A^{T} W A$ is symmetric positive definite square matrix.

- Exploit sparsity of $A^{T} W A$.

- Use QR or LU or LDL decomposition for fast inversion, instead of SVD decomposition.

- Use a parallelizable solver.

- Estimate deformation, topographic error.

- Estimate variance of the estimates given by diagonal of: inverse $\left(A^{T} W A\right)$, which is a non-sparse matrix. For this purpose, use a selected parallel inversion method based on LDL decomposition.

\section{EXPERIMENTAL RESULTS}

The proposed algorithms are tested on three ERS-1/2 stacks of Germany. Fig. 2, Fig. 3 and Fig. 4 show the conventional reference network and the improved reference network for these three different test cases respectively. In each of these figures, the conventional reference network is shown in upperleft part. Various clusters are visible due to low density of PSs in rural areas. Variance of PS deformation velocity of main cluster of conventional reference network is displayed in lower-left. As can be seen, the variance is high in regions that are not connected properly. The improved reference network is shown in upper-right and it is a single-network. Variance of PS deformation velocity of improved reference network is displayed in lower-right and a clear improvement is visible.

\section{CONCLUSION}

PSI is a powerful tool for long-term deformation mapping, and improving the reference network has potential to enhance the deformation velocity maps. Wide-area PSI, wherein, urban and non-urban areas are processed together, would benefit from this. The future scope of the proposed algorithms is especially for Sentinel-1 data.

\section{REFERENCES}

[1] R. Bamler and P. Hartl, "Synthetic aperture radar interferometry- Topical review," Inverse problems- 14, R1 R54, IOP Publishing Limited (UK), 1998.

[2] P. A. Rosen, S. Hensley, I. R. Joughin, F. K. Li, S. N. Madsen, E. Rodriguez, and R. M. Goldstein, "Synthetic Aperture Radar Interferometry," In: Proc. IEEE, 88(3): 333382, 2000.

[3] A. Ferretti, C. Prati, and F. Rocca, "Permanent scatterers in SAR interferometry," IEEE TGARS, vol. 39, No. 1, pp. 8-20, 2001.

[4] A. Ferretti, C. Prati, and F. Rocca, "Nonlinear subsidence rate estimation using permanent scatterers in differential SAR interferometry," IEEE TGARS, vol. 38, no. 5, pp. 2202-2212, 2000.

[5] P. Berardino, G. Fornaro, R. Lanari, and E. Sansosti, “A new algorithm for surface deformation monitoring based on small baseline differential SAR interferograms," IEEE TGARS, vol. 40, no. 11, p. 2375, 2002.

[6] K. Goel, and N. Adam, "An advanced algorithm for deformation estimation in non-urban areas," ISPRS Journal of Photogrammetry and Remote Sensing, vol. 73, pp. 100-110, 2012.

[7] A. Ferretti, A. Fumagalli, F. Novali, C. Prati, F. Rocca, and A. Rucci, "A New Algorithm for processing interferometric data-stacks: SqueeSAR," IEEE TGARS, vol. 49, no. 9, p. 3460, 2011. 

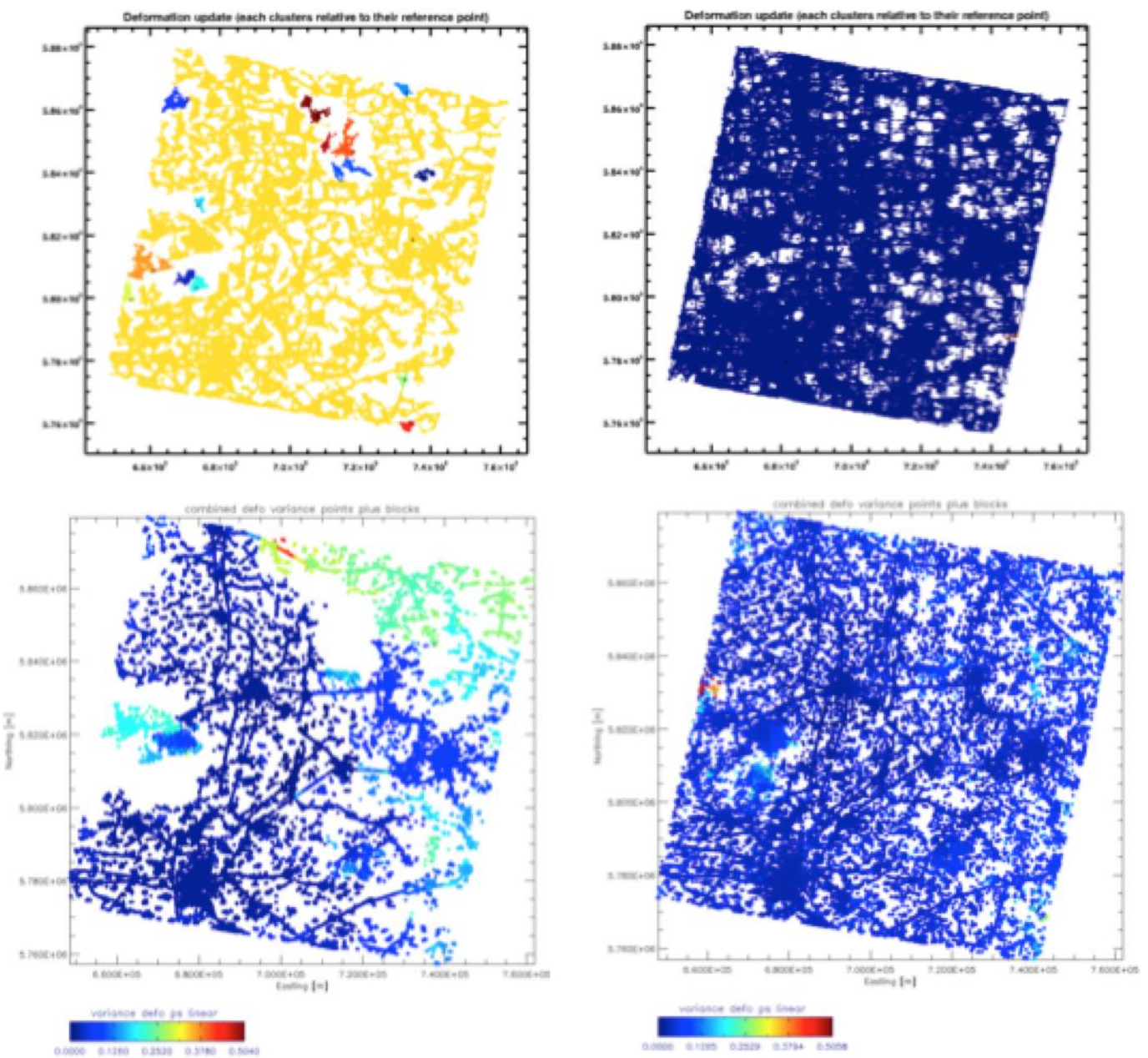

Figure 2: Experimental results for first ERS-1/2 stack of Germany. Conventional reference network divided into clusters due to low density of PSs in rural areas (upper-left). Variance of PS deformation velocity of main cluster of conventional reference network (lower-left). Improved reference network (upper-right). Variance of PS deformation velocity of improved reference network (lower-right).
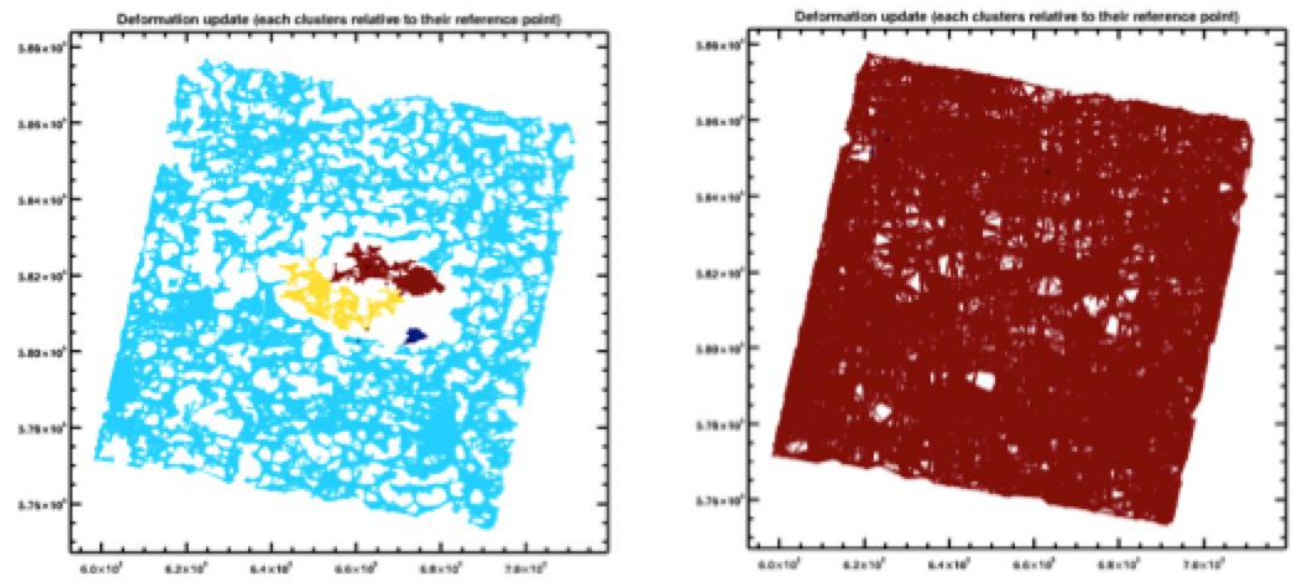

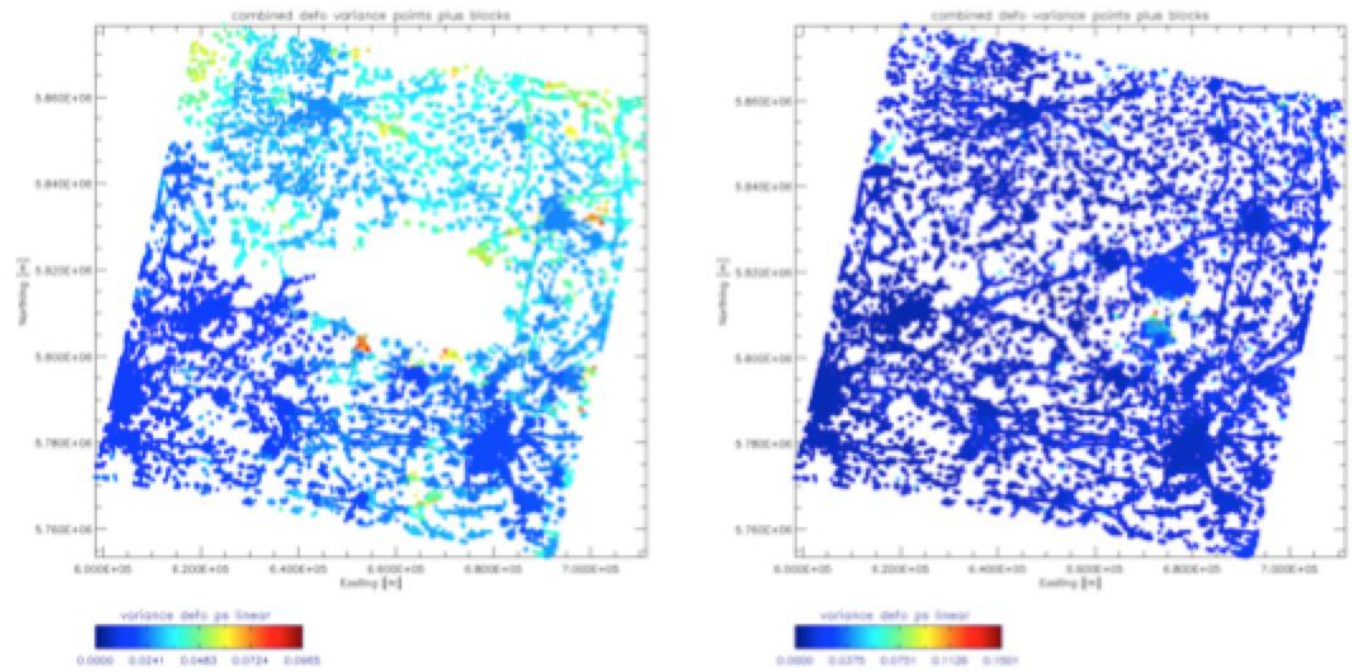

Figure 3: Experimental results for second ERS-1/2 stack of Germany. Conventional reference network divided into clusters due to low density of PSs in rural areas (upper-left). Variance of PS deformation velocity of main cluster of conventional reference network (lower-left). Improved reference network (upper-right). Variance of PS deformation velocity of improved reference network (lower-right).
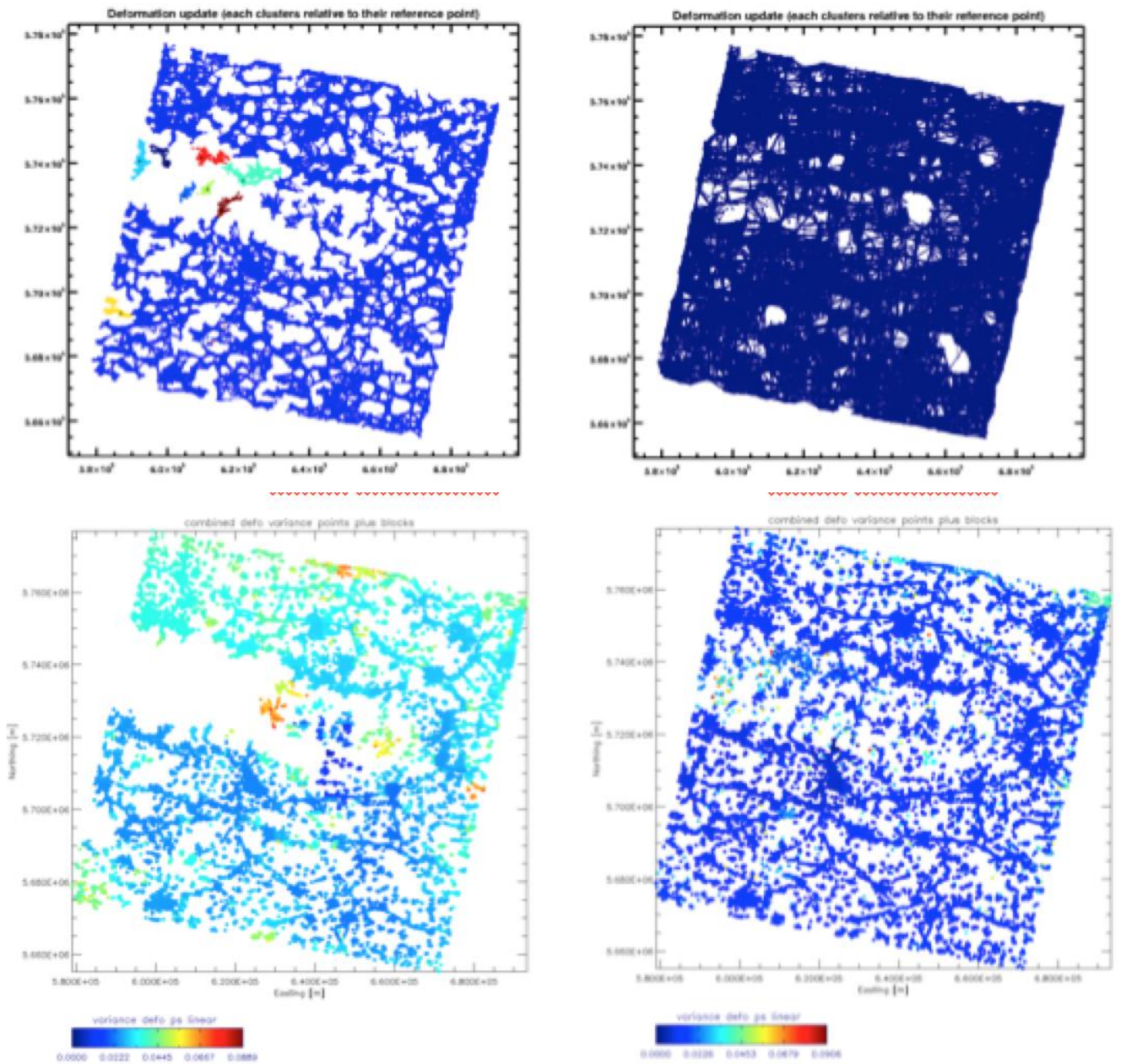

Figure 4: Experimental results for third ERS-1/2 stack of Germany. Conventional reference network divided into clusters due to low density of PSs in rural areas (upper-left). Variance of PS deformation velocity of main cluster of conventional reference network (lower-left). Improved reference network (upper-right). Variance of PS deformation velocity of improved reference network (lower-right). 